\title{
Ground States of Quantum Spin Systems
}

\author{
Ola Bratteli ${ }^{1 \star}$, Akitaka Kishimoto ${ }^{1 \star \star}$, and Derek W. Robinson ${ }^{2}$ \\ 1 Centre de Physique Théorique II, CNRS, F-13288 Marseille Cedex 2, France \\ 2 Department of Mathematics, University of N.S.W., Sydney, Australia, and \\ Centre de Physique Théorique II, CNRS, F-13288 Marseille Cedex 2, France
}

\begin{abstract}
We prove that ground states of quantum spin systems are characterized by a principle of minimum local energy and that translationally invariant ground states are characterized by the principle of minimum energy per unit volume.
\end{abstract}

\section{Introduction}

Let $\tau$ be a strongly continuous one-parameter group of *-automorphisms of a $C^{*}$-algebra $\mathfrak{A}$. Denote the generator of $\tau$ by $\delta$. A state $\omega$ over $\mathfrak{A}$ is defined to be a $\tau$-ground state if

$$
-i \omega\left(A^{*} \delta(A)\right) \geqq 0
$$

for all $A$ in the domain $D(\delta)$ of $\delta$. It follows that $\omega$ is $\tau$-invariant and hence generates a covariant representation $\left(\mathscr{H}_{\omega}, \Pi_{\omega}, U_{\omega}, \Omega_{\omega}\right)$ of $(\mathfrak{H}, \tau)$. If $U_{\omega}$ is chosen such that $U_{\omega}(t) \Omega_{\omega}=\Omega_{\omega}$ and if $H_{\omega}$ is the infinitesimal generator of $U_{\omega}$ then the ground state condition implies $H_{\omega} \geqq 0$. More generally a state $\omega$ is a $\tau$-ground state if, and only if, $\omega$ is $\tau$-invariant and $H_{\omega} \geqq 0$. (For further details see [1], Chapter V).

A state $\omega$ is called a $(\tau, \beta)$-KMS state, for $\beta \in \mathbb{R}$, if

$$
\omega(A B)=\omega\left(B \tau_{i \beta}(A)\right)
$$

for all entire analytic elements $A, B$ of $\tau$. The $(\tau, \beta)$-KMS states arise from the Gibbs formalism of equilibrium statistical mechanics and correspond to equilibrium states at inverse temperature $\beta$. The ground states correspond to the zero temperature states.

One can prove, for example by the Sewell condition [2] (see also [3]) characterizing $(\tau, \beta)$-KMS states, that if $\omega_{\beta}$ is a family of $(\tau, \beta)$-KMS states which is weak*-convergent as $\beta \rightarrow \infty$ then the limit state $\omega$ is a $\tau$-ground state. For this reason ground states are sometimes referred to as $(\tau,+\infty)$-KMS states. This

* Present address : Department of Mathematics, University of Oslo, Blindern, Oslo 3, Norway

$\star \star$ Present address: Department of Mathematics, University of Ottawa, Ontario, Canada K1N 9B4 
notation is somewhat misleading because several properties of $(\tau, \beta)$-KMS states and ground states are quite different.

The simplest interesting example is the one-dimensional Ising model with translationally invariant nearest neighbour interaction and with zero external field. This model has a unique $(\tau, \beta)$-KMS state $\omega_{\beta}$ for all $\beta \in \mathbb{R}$ and $\omega_{\beta}$ is automatically translationally invariant. But there are two extremal translationally invariant $\tau$-ground states $\omega_{ \pm}, \omega_{+}$corresponds to all spins up and $\omega_{-}$to all spins down. One has $\omega_{\beta} \rightarrow\left(\omega_{+}+\omega_{-}\right) / 2$ as $\beta \rightarrow \infty$. Moreover there are an infinite number of non translationally invariant ground states. A typical such state $\omega_{x}$ has all spins to the left of $x \in \mathbb{Z}$ up and all remaining spins down. These states, $\omega_{x}$, are all pure and as $\omega_{x_{1}}$, and $\omega_{x_{2}}$ differ only by the reversal of a finite number of spins they are equivalent, i.e. they generate unitarily equivalent representations. For finite $\beta$, however, two extremal $(\tau, \beta)$-KMS states are either equal or disjoint. Thus parity, multiplicity, and equivalence properties of finite temperature KMS states and ground states all differ for this model.

Nevertheless ground states and KMS states do have many common properties of stability. In [4] we analyzed various forms of dynamical stability of these sets of states and in this note we examine two properties of kinematical stability for spin systems.

Araki and Sewell [5], [2] have shown that if $\tau$ is the dynamical group of a quantum spin system generated by an interaction $\Phi$ then the $(\tau, \beta)$-KMS states are exactly those states $\omega$ which satisfy

$$
\tilde{S}_{\Lambda}(\omega)-\beta \omega\left(\tilde{H}_{\Phi}(\Lambda)\right)=\sup _{\omega^{\prime} \in C_{\Lambda}^{\omega}}\left\{\tilde{S}_{\Lambda}\left(\omega^{\prime}\right)-\beta \omega^{\prime}\left(\tilde{H}_{\Phi}(\Lambda)\right)\right\}
$$

for all $\Lambda$ where

$$
C_{\Lambda}^{\omega}=\left\{\omega^{\prime} ; \omega^{\prime}\left|\mathfrak{U}_{A^{c}}=\omega\right| \mathfrak{U}_{A^{c}}\right\}
$$

Here $\tilde{S}_{\Lambda}$ denotes the conditional entropy of the finite set $\Lambda$ and $\tilde{H}_{\Phi}(\Lambda)$ the conditional energy,

$$
\tilde{H}_{\Phi}(\Lambda)=\sum_{X \cap \Lambda \neq \phi} \Phi(X)
$$

(we use the standard notation for spin systems, see, for example, [6]). We prove the zero temperature analogue of this result, i.e. $\omega$ is a $\tau$-ground state if and only if

$$
\omega\left(\tilde{H}_{\Phi}(\Lambda)\right)=\inf _{\omega^{\prime} \in C_{\Lambda}^{\omega}} \omega^{\prime}\left(\tilde{H}_{\Phi}(\Lambda)\right)
$$

for all $\Lambda$. We then deduce for the lattice $L=\mathbb{Z}^{v}$, and $\mathbb{Z}^{v}$-invariant interactions $\Phi$, that a state is a $\mathbb{Z}^{\nu}$-invariant $\tau$-ground state if, and only if, it minimizes the energy per unit volume. This last characterization is the zero temperature analogue of a result of Lanford-Robinson [7] and Araki [8]. Ruelle has earlier proved the ifpart of this result, [9].

\section{Local Stability}

The main result of this note is the following theorem whose assumptions are stated in a somewhat implicit form. It is known that they are satisfied for all interactions 
$\Phi$ such that

$$
\sum_{n \geqq 1} e^{\lambda n} \sup _{x \in L} \sum_{\substack{X \ni x \\|X|=n+1}}\|\Phi(X)\|<+\infty
$$

for some $\lambda>0$ (see [6]), and they are also satisfied if there exists an increasing sequence $\Lambda_{n}$ of bounded subsets of $\mathbb{Z}^{v}$ such that

$$
\left\|\Sigma\left\{\Phi(X) ; X \cap \Lambda_{n} \neq \phi, X \cap \Lambda_{n+m}^{c} \neq \phi\right\}\right\| \leqq K n e^{-\lambda m}
$$

for some $K, \lambda>0$ (see [14]).

Theorem 1. Let $\mathfrak{A}=\overline{\bigcup_{A} \mathfrak{H}_{A}}$ be the $C^{*}$-algebra of a quantum spin system and let $\tau$ be an automorphism group of $\mathfrak{A}$ such that $\mathscr{D}=\bigcup_{\Lambda} \mathfrak{H}_{A}$ is a core for the generator $\delta$ of $\tau$. For each finite $\Lambda \subset L$, let $\tilde{H}(\Lambda)=\tilde{H}(\Lambda)^{*} \in \mathfrak{H}$ be elements such that

$$
\delta(A)=i[\tilde{H}(\Lambda), A]
$$

for all $A \in \mathfrak{U}_{\Lambda}$.

The following conditions are equivalent for a state $\omega \in E_{\mathfrak{Q}}$

1. $\omega(\tilde{H}(\Lambda))=\inf _{\omega^{\prime} \in C_{\Lambda}^{()}} \omega^{\prime}(\tilde{H}(\Lambda))$

for all $\Lambda \subset L$, where

$$
C_{\Lambda}^{\omega}=\left\{\omega^{\prime} ; \omega^{\prime} \in E_{\mathfrak{Q}}, \omega^{\prime}\left|\mathfrak{U}_{A^{c}}=\omega\right| \mathfrak{U}_{A^{c}}\right\}
$$

2. $\omega$ is a $\tau$-ground state, i.e.

$$
-i \omega\left(A^{*} \delta(A)\right) \geqq 0
$$

for all $A \in D(\delta)$.

Remark. The existence of elements $\tilde{H}(\Lambda)=\tilde{H}(\Lambda)^{*}$ with the given properties follows from [15].

Proof. $1 \Rightarrow 2$. This is proved in a similar fashion to the finite temperature analogue, [2], [5]. If $A=A^{*} \in \mathfrak{H}_{A}$ then Condition 1 implies that

$$
\omega\left(e^{i t A} \tilde{H}(\Lambda) e^{-i t A}\right) \geqq \omega(\tilde{H}(\Lambda))
$$

and by differentiation at $t=0$ one deduces that

$$
-\omega(\delta(A))=\omega(i[A, \tilde{H}(\Lambda)])=0
$$

and as $\mathscr{D}$ is a core for $\delta$, it follows that $\omega$ is $\tau$-invariant.

Now consider the operator $\gamma_{B}: \mathfrak{U} \mapsto \mathfrak{U}$, defined by

$$
\gamma_{B}(A)=B^{*} A B-\left\{B^{*} B, A\right\} / 2 .
$$

We argue as in [2] that if $B \in \mathfrak{U}_{A}$, and $T_{t}=\exp \left\{t \gamma_{B}\right\}$, then $T_{t}^{*} E_{\mathfrak{I}} \subseteq E_{\mathscr{N}}$ and $T_{t}^{*} \omega \in C_{A}^{\omega}$ for all $t \in \mathbb{R}_{+}$. Hence it follows from Condition 1 that

$$
T_{t}^{*} \omega(\tilde{H}(\Lambda)) \geqq \omega(\tilde{H}(\Lambda))
$$


and thus

$$
\omega\left(\gamma_{B}(\tilde{H}(\Lambda))\right) \geqq 0
$$

But the $\tau$-invariance of $\omega$ implies that

$$
-i \omega\left(B^{*} \delta(B)\right)=\omega\left(\gamma_{B}(\tilde{H}(\Lambda))\right)
$$

and hence

$$
-i \omega\left(B^{*} \delta(B)\right) \geqq 0
$$

Since $\mathscr{D}$ is a core for $\delta$ it follows that $\omega$ is a ground state.

$2 \Rightarrow 1$. Since there is no appropriate analogue of the Gibbs condition for ground states, we have to proceed differently from [2], [5]. To this end we need a result on extensions of normal states to semibounded self-adjoint elements affiliated with a von Neumann algebra.

The following lemma is for convenience formulated for positive operators, but it extends with trivial modifications to semi-bounded operators. It is a special case of a result of [10] but we nevertheless include a proof for completeness.

Lemma 1. Let $\mathfrak{M}$ be a von Neumann algebra on a Hilbert space $\mathscr{H}$, let $A$ be a positive, self-adjoint operator affiliated with $\mathfrak{M}$. Let $\omega$ be a normal state on $\mathfrak{M}$, and $\left\{\xi_{n}\right\}_{n \geqq 1}$ a sequence of vectors in $\mathscr{H}$ such that

$$
\omega=\sum_{n \geqq 1} \omega_{\xi_{n}}, \quad \sum_{n \geqq 1}\left\|\xi_{n}\right\|^{2}=1
$$

The following conditions are equivalent

1. $\sup \left\{\omega(B) ; B \in \mathfrak{M}_{+}, B \leqq A\right\}<+\infty$

2. $\xi_{n} \in D\left(A^{\frac{1}{2}}\right)$ for all $n$ and

$$
\sum_{n \geqq 1}\left\|A^{\frac{1}{2}} \xi_{n}\right\|^{2}<+\infty
$$

If these conditions are fulfilled, then

$$
\sup \left\{\omega(B) ; B \in \mathfrak{M}_{+}, B \leqq A\right\}=\sum_{n \geqq 1}\left\|A^{\frac{1}{2}} \xi_{n}\right\|^{2}
$$

Definition. In the situation covered by Lemma 1, we define

$$
\omega(A)=\sum_{n \geqq 1}\left\|A^{\frac{1}{2}} \xi_{n}\right\|^{2}=\sup \left\{\omega(B) ; B \in \mathfrak{M}_{+}, B \leqq A\right\}
$$

if the conditions of the Lemma are fulfilled, and

$$
\omega(A)=+\infty
$$

otherwise.

Proof. Define

$$
\begin{aligned}
& K_{1}=\sup \left\{\omega(B) ; B \in \mathfrak{M}_{+}, B \leqq A\right\} \\
& K_{2}=\sum_{n \geqq 1}\left\|A^{\frac{1}{2}} \xi_{n}\right\|^{2}
\end{aligned}
$$


whenever the right hand sides exist, and define $K_{1}, K_{2}$ to be equal to $+\infty$ otherwise.

Let $f_{n}$ be the function defined on $\mathbb{R}_{+}$by

$$
f_{n}(x)=\left\{\begin{array}{lll}
x & \text { if } & 0 \leqq x \leqq n \\
n & \text { if } & x>n
\end{array}\right.
$$

It follows that $A_{n}=f_{n}(A) \in \mathfrak{M}$ for $n=1,2, \ldots$ and by spectral theory it follows that the quadratic forms defined by $A_{n}$ converges monotonically to the form defined by $A$, i.e. if $\xi \in \mathscr{H}$ then

$$
\left(\xi, A_{n} \xi\right) \rightarrow \begin{cases}\left(A^{\frac{1}{2}} \xi, A^{\frac{1}{2}} \xi\right) & \text { if } \xi \in D\left(A^{\frac{1}{2}}\right) \\ +\infty & \text { otherwise }\end{cases}
$$

As a consequence of this and the monotone convergence theorem

$$
\lim _{n \rightarrow \infty} \sum_{k \geqq 1}\left(\xi_{k}, A_{n} \xi_{k}\right)=K_{2}
$$

Hence

$$
K_{2} \leqq K_{1}
$$

But conversely, if $B \in \mathfrak{M}_{+}$and $B \leqq A$ as form, then

$$
\omega(B)=\sum_{k \geqq 1}\left(\xi_{k}, B \xi_{k}\right) \leqq \sum_{k \geqq 1}\left(A^{\frac{1}{2}} \xi_{k}, A^{\frac{1}{2}} \xi_{k}\right)=K_{2}
$$

and hence

$$
K_{1} \leqq K_{2}
$$

Next we need a result on quasi-equivalence of states which coincide outside of a finite subset of the lattice. This is covered by the following

Lemma 2. Let $\mathfrak{A}$ be a $C^{*}$-algebra of the form

$$
\mathfrak{U}=M_{n} \otimes \mathfrak{A}_{0}
$$

where $M_{n}$ is the full complex $n \times n$ matrix algebra and $\mathfrak{X}_{0}$ is an arbitrary $C^{*}$-algebra. Let $\omega_{1}$ and $\omega_{2}$ be states on $\mathfrak{A}$ such that

$$
\omega_{1}\left|\mathfrak{U}_{0}=\omega_{2}\right| \mathfrak{U}_{0}
$$

It follows that $\omega_{1}$ and $\omega_{2}$ are quasi-equivalent.

Proof. Let $\mathfrak{A}^{\prime \prime}$ be the von Neumann enveloping algebra of $\mathfrak{A}$, and let $\omega_{1}, \omega_{2}$ also denote the normal extension of $\omega_{1}, \omega_{2}$ to $\mathfrak{U}^{\prime \prime}$. Further let $C$ be an element of the center of $\mathfrak{A}^{\prime \prime}$, and pick a net $\left\{A_{\alpha}\right\}$ in $\mathfrak{U}$ converging to $C$ in the weak*-topology on $\mathfrak{U}^{\prime \prime}$. If $\left\{E_{i j}\right\}_{1 \leqq i, j \leqq n}$ are matrix units for $M_{n}$ then

$$
A \in \mathfrak{A} \mapsto E(A)=\sum_{i=1}^{n} E_{i 1} A E_{1 i}
$$


is a projection of norm one from $\mathfrak{A}$ onto $\mathfrak{A}_{0}=M_{n}^{\prime} \cap \mathfrak{U}$, [11]. Since $C$ is a central element it follows that

$$
\begin{aligned}
\lim _{\alpha} E\left(A_{\alpha}\right) & =\sum_{i=1}^{n} \lim _{\alpha} E_{i 1} A_{\alpha} E_{1 i} \\
& =\sum_{i=1}^{n} E_{i 1} C E_{1 i} \\
& =\sum_{i=1}^{n} E_{i i} C=C
\end{aligned}
$$

But as $E\left(A_{\alpha}\right) \in \mathfrak{H}_{0}$ we have

$$
\begin{aligned}
\omega_{1}(C) & =\lim _{\alpha} \omega_{1}\left(E\left(A_{\alpha}\right)\right) \\
& =\lim _{\alpha} \omega_{2}\left(E\left(A_{\alpha}\right)\right)=\omega_{2}(C)
\end{aligned}
$$

Thus the restrictions of $\omega_{1}$ and $\omega_{2}$ to the center of $\mathfrak{U}^{\prime \prime}$ coincide, and the two states have the same central support. It follows that $\omega_{1}$ and $\omega_{2}$ are quasi-equivalent.

We now finish the proof of the implication $1 \Rightarrow 2$ in the theorem. Assume that $\omega$ is a ground state, and let $\omega$ also denote the normal extension of $\omega$ to $\mathfrak{M}_{\omega}$ $=\Pi_{\omega}(\mathfrak{U})^{\prime \prime}$. The generator $H_{\omega}$ of the canonical unitary group defined by $\tau$ on $\mathscr{H}_{\omega}$ is positive, and $H_{\omega}$ is affiliated with $\mathfrak{M}_{\omega}$ by $[12,13]$.

Note that

$$
i\left[H_{\omega}-\Pi_{\omega}(\tilde{H}(A)), \Pi_{\omega}(A)\right] \psi=\left(\Pi_{\omega}(\delta(A))-\Pi_{\omega}(\delta(A))\right) \psi=0
$$

for $A \in \mathfrak{A}_{\Lambda}$, and $\psi \in D\left(H_{\omega}\right)$.

Thus if $U_{\Lambda}(t)=\exp \left\{i t\left(H_{\omega}-\Pi_{\omega}(\tilde{H}(\Lambda))\right)\right\}$ one has

$$
\begin{aligned}
& U_{\Lambda}(t) \Pi_{\omega}(A) U_{\Lambda}(t)^{*} \psi=\Pi_{\omega}(A) \psi \\
& \quad+i \int_{0}^{t} d s U_{\Lambda}(s)\left[H_{\omega}-\Pi_{\omega}(\tilde{H}(A)), \Pi_{\omega}(A)\right] U_{s}(s)^{-1} \psi \\
& =\Pi_{\omega}(A) \psi
\end{aligned}
$$

for all $A \in \mathfrak{U}_{A}$, and $\psi \in D\left(H_{\omega}\right)$. Thus $U_{A}(t) \in \Pi_{\omega}\left(\mathfrak{U}_{\Lambda}\right)^{\prime}$. But the Trotter product formula,

$$
U_{\Lambda}(t)=\mathrm{s} \cdot \lim _{n \rightarrow \infty}\left(U_{\omega}(t / n) e^{-i t \Pi_{\omega}(\tilde{H}(A)) / n}\right)^{n}
$$

shows that $U_{\Lambda}(t) \in \Pi_{\omega}(\mathfrak{H})^{\prime \prime}$. Thus $U_{\Lambda}(t) \in \Pi_{\omega}\left(\mathfrak{U}_{\Lambda}\right)^{\prime} \cap \Pi_{\omega}(\mathfrak{H})^{\prime \prime}$ and $H_{\omega}-\Pi_{\omega}(\tilde{H}(\Lambda))$ is affiliated with $\Pi_{\omega}\left(\mathfrak{U}_{\Lambda}\right)^{\prime} \cap \Pi_{\omega}(\mathfrak{U})^{\prime \prime}$. But it follows from [11] that

$$
\Pi_{\omega}\left(\mathfrak{U}_{\Lambda}\right)^{\prime} \cap \Pi_{\omega}(\mathfrak{A})^{\prime \prime}=\Pi_{\omega}\left(\mathfrak{U}_{A^{c}}\right)^{\prime \prime} .
$$

Now if $\omega^{\prime}$ is a state on $\mathfrak{U}$ such that

$$
\omega^{\prime}\left|\mathfrak{A}_{\Lambda^{c}}=\omega\right| \mathfrak{A}_{\Lambda^{c}},
$$

Lemma 2 implies that $\omega^{\prime}$ extends to a normal state of $\mathfrak{M}_{\omega}$, and hence $\omega^{\prime}\left(H_{\omega}\right)$ is well defined. Furthermore the positivity of $H_{\omega}$ implies

$$
0=\omega\left(H_{\omega}\right)=\inf _{\omega^{\prime} \in C_{\Lambda}^{\omega}} \omega^{\prime}\left(H_{\omega}\right) .
$$

Also, since by continuity $\omega\left|\Pi_{\omega}\left(\mathfrak{U}_{A^{c}}\right)^{\prime \prime}=\omega^{\prime}\right| \Pi_{\omega}\left(\mathfrak{H}_{A^{c}}\right)^{\prime \prime}$, it follows from the second characterization in the definition of $\omega^{\prime}\left(H_{\omega}\right)$ that

$$
\omega\left(H_{\omega}-\tilde{H}(\Lambda)\right)=\omega^{\prime}\left(H_{\omega}-\tilde{H}(\Lambda)\right) .
$$


In particular $\omega^{\prime}\left(H_{\omega}\right)<+\infty$, and the first characterization of $\omega^{\prime}\left(H_{\omega}\right)$ implies $\omega^{\prime}\left(H_{\omega}\right)-\omega^{\prime}\left(H_{\omega}-\tilde{H}(\Lambda)\right)=\omega^{\prime}(\tilde{H}(\Lambda))$.

It follows that

$$
\begin{aligned}
\omega(\tilde{H}(\Lambda)) & =\omega\left(H_{\omega}\right)-\omega\left(H_{\omega}-\tilde{H}(\Lambda)\right) \\
& =\inf _{\omega^{\prime} \in C_{\Lambda}^{\omega}}\left\{\omega^{\prime}\left(H_{\omega}\right)-\omega^{\prime}\left(H_{\omega}-\tilde{H}(\Lambda)\right)\right\} \\
& =\inf _{\omega^{\prime} \in C_{\Lambda}^{\omega}} \omega^{\prime}(\tilde{H}(\Lambda)) .
\end{aligned}
$$

\section{Invariant States}

Next assume that the spin system is on the lattice $\mathbb{Z}^{v}$. The group $\mathbb{Z}^{v}$ has an action $\alpha$ on the quantum spin algebra $\mathfrak{A}$ and the interaction $\Phi$ is $\mathbb{Z}^{v}$-invariant if

$$
\Phi(X+x)=\alpha_{x}(\Phi(X))
$$

for all $x \in \mathbb{Z}^{v}$, and $X \subset \mathbb{Z}^{v}$. If $H_{\Phi}(\Lambda)$ is the local energy associated with $\Phi$,

$$
H_{\Phi}(\Lambda)=\sum_{x \subset \Lambda} \Phi(X)
$$

one can define a mean energy functional over the set of $\mathbb{Z}^{v}$-invariant states $E_{\mathfrak{U}}^{\mathbb{Z}^{v}}$ by

$$
\omega \in E_{\mathfrak{U}}^{\mathbb{Z}^{v}} \mapsto H_{\Phi}(\omega)=\lim _{\Lambda \rightarrow \infty} \omega\left(H_{\Phi}(\Lambda)\right) /|\Lambda|
$$

whenever

$$
\|\Phi\|=\sum_{X \ni 0}\|\Phi(x)\| /|x|<+\infty .
$$

One has

$$
H_{\Phi}(\omega)=\sum_{X \ni 0} \omega(\Phi(X)) /|X|
$$

and hence $\left|H_{\Phi}(\omega)\right| \leqq\|\Phi\|$.

Under slightly more stringent conditions on $\Phi$ one can deduce that the $\mathbb{Z}^{\prime}$ invariant ground states are the states which minimize $H_{\Phi}$.

Theorem 2. Let $\Phi$ be a $\mathbb{Z}^{v}$-invariant interaction such that

$$
\|\Phi\|_{\lambda}=\sum_{X \ni 0}\|\Phi(X)\| e^{\lambda|X|}<+\infty
$$

for some $\lambda>0$ and let $\tau^{\Phi}$ denote the associated dynamical group.

If $\omega$ is a $\mathbb{Z}^{v}$-invariant state the following are equivalent

1. $\omega$ is a $\tau^{\Phi}$-ground state,

2. $\omega$ minimizes $H_{\Phi}$.

Proof. $1 \Rightarrow 2$. Let $\omega$ be a $\mathbb{Z}^{v}$-invariant $\tau^{\Phi}$-ground state. Theorem 1 implies that

$$
\omega\left(\tilde{H}_{\Phi}(\Lambda)\right) \leqq \omega^{\prime}\left(\tilde{H}_{\Phi}(\Lambda)\right)
$$

for all $\Lambda \subset \mathbb{Z}^{v}$ and all $\omega^{\prime} \in C_{\Lambda}^{\omega}$ with

$$
\tilde{H}_{\Phi}(\Lambda)=\sum_{X \cap \Lambda \neq \phi} \Phi(X) \text {. }
$$

Now let $\sigma$ be a $\mathbb{Z}^{v}$-invariant state and define $\omega^{\prime}$ by $\omega^{\prime}=\sigma\left|\mathfrak{U}_{\Lambda} \otimes \omega\right| \mathfrak{U}_{\Lambda^{c}}$. 
Thus $\omega^{\prime} \in C_{\Lambda}^{\omega}$ and

$$
\omega\left(\tilde{H}_{\Phi}(\Lambda)\right) \leqq \omega^{\prime}\left(\tilde{H}_{\Phi}(\Lambda)\right) .
$$

But $\sigma\left(H_{\Phi}(\Lambda)\right)=\omega^{\prime}\left(H_{\Phi}(\Lambda)\right)$ and hence

$$
\begin{aligned}
\omega\left(H_{\Phi}(\Lambda)\right) & \leqq \sigma\left(H_{\Phi}(\Lambda)\right)+\omega^{\prime}\left(W_{\Phi}(\Lambda)\right)-\omega\left(W_{\Phi}(\Lambda)\right) \\
& \leqq \sigma\left(H_{\Phi}(\Lambda)\right)+2\left\|W_{\Phi}(\Lambda)\right\|
\end{aligned}
$$

with $W_{\Phi}(\Lambda)=\tilde{H}_{\Phi}(\Lambda)-H_{\Phi}(\Lambda)$. Dividing both sides by $|\Lambda|$ and using

$$
\lim _{\Lambda \rightarrow \infty}\left\|W_{\Phi}(\Lambda)\right\| /|\Lambda|=0
$$

where $\Lambda \rightarrow \infty$ in the sense of van Hove, one finds

$$
H_{\Phi}(\omega) \leqq H_{\Phi}(\sigma) \text {. }
$$

Thus

$$
H_{\Phi}(\omega)=\inf _{\sigma \in E^{\mathbb{Z}^{v}}} H_{\Phi}(\sigma)
$$

where $E_{\mathfrak{Q}}^{\mathbb{Z}^{v}}$ is the set of $\mathbb{Z}^{v}$-invariant states over $\mathfrak{U}$.

$2 \Rightarrow 1$. This has already been proved by Ruelle [9].

\section{References}

1. Bratteli,O., Robinson,D.W.: Operator algebras and quantum statistical mechanics. Berlin, Heidelberg, New York: Springer (to appear)

2. Sewell, G.L. : KMS conditions and local thermodynamic stability. II. Commun. math. Phys. 55, 53 (1977)

3. Araki,H.: On $K M S$ states of a $C^{*}$-dynamical system, in $C^{*}$-algebras and applications to physics. Lecture notes in mathematics, Vol. 650. Berlin, Heidelberg, New York: Springer 1978

4. Bratteli,O., Kishimoto,A., Robinson,D.W.: Stability properties and the KMS condition. Commun. math. Phys. 61, 209 (1978)

5. Araki,H., Sewell, G.L.: KMS conditions and local thermodynamic stability of quantum lattice systems. Commun. math. Phys. 52, 103 (1977)

6. Ruelle,D.: Statistical mechanics. New York-Amsterdam: Benjamin 1969

7. Lanford,O.E., Robinson,D.W.: Statistical mechanics of quantum spin systems. III. Commun. math. Phys. 9, 327 (1968)

8. Araki,H.: On the equivalence of the KMS condition and the variational principle for quantum lattice systems. Commun. math. Phys. 38, 1 (1974)

9. Ruelle,D.: Some remarks on the ground state of infinite systems in statistical mechanics. Commun. math. Phys. 11, 339 (1968)

10. Bratteli,O.: Conservation of estimates in quantum field theory. Commun. Pure Appl. Math. 25, 759 (1972)

11. Powers, R.: Representations of uniformly hyperfinite algebras and their associated von Neumann rings. Ann. Math. 86, 138 (1967)

12. Borchers, H.: Energy and momentum as observables in quantum field theory. Commun. math. Phys. 2, 49 (1966)

13. Arveson, W.: On groups of automorphisms of operator algebras. J. Funct. Anal. 15, 217 (1974)

14. Bratteli,O., Kishimoto,A.: Generation of semi-groups, and two-dimensional quantum lattice systems. J. Funct. Anal. (to appear)

15. Elliott, G.: Derivations of matroid $C^{*}$-algebras. Inventiones Math. 9, 253 (1970)

Communicated by H. Araki

Received July 24, 1978 